\title{
Control Law Design for Discrete Linear Repetitive Processes with non-local Updating Structures
}

\author{
Błażej Cichy • Krzysztof Gałkowski • \\ Eric Rogers • Anton Kummert
}

Received: date / Accepted: date

\begin{abstract}
Repetitive processes are a class of 2D systems where information propagation in one direction is of finite duration. These processes make a series of sweeps, termed passes, through a set of dynamics and on completion of each pass resetting to the starting position occurs ready for the start of the next pass. The control problem is that the previous pass output, termed the pass profile, acts as a forcing function on the current pass and can result in oscillations that increase in amplitude from pass-to-pass. In the case of discrete dynamics, these processes have structural links with $2 \mathrm{D}$ systems described by the well known Roesser and Fornasini-Marchesini state-space models but some applications require updating structures that cannot be represented by these models. This requirement arises either in adequately modeling the dynamics or as a result of the control law structure and requires the development of a systems theory for eventual use in applications. In this paper such a theory is advanced through the development of new control law design algorithms.
\end{abstract}

\section{Introduction}

Repetitive processes make a series of sweeps, termed passes, through a set of dynamics defined over a finite duration known as the pass length. Once a pass is

This work is partially supported by National Science Centre in Poland under grant No. 2011/01/B/ST7/00475.

B. Cichy and K. Gałkowski

Institute of Control and Computation Engineering, University of Zielona Góra, ul. Podgórna 50, 65-246 Zielona Góra, Poland. Tel.: +48-68-328-2422, Fax: +48-68-328-751.

E-mail: $\{$ B.Cichy,K.Galkowski\}@issi.uz.zgora.pl

E. Rogers

Electronics and Computer Science, University of Southampton, Southampton SO17 1BJ, UK.

E-mail: etar@ecs.soton.ac.uk

A. Kummert

Faculty of Electrical, Information and Media Engineering, University of Wuppertal, Riener Gruenter-Strasse 21, D-42119, Wuppertal, Germany. E-mail: kummert@uni-wuppertal.de 
completed the process resets to the initial position and the next one commences. Each pass profile acts as a disturbance function on, and hence contributes to, the dynamics of the next one [Rogers et al (2007)]. This interaction between successive pass profiles can result in oscillations that increase in amplitude in the pass-topass direction and the core requirement for any control law is to prevent such oscillations from arising and hence facilitate tracking of a reference signal.

Consider the case of discrete dynamics and let $y_{k}(p), 0 \leq p \leq \alpha-1, k \geq 0$, denote the, scalar or vector valued, pass profile which is of finite duration $\alpha$. Then in a repetitive process $y_{k}(p)$ acts as a forcing function on, and hence contributes to, the dynamics of the next pass profile $y_{k}(p), k \geq 0$.

Repetitive processes have their origins in coal mining [Rogers et al (2007)] where in the longwall mode of operation coal is extracted by a series of passes of the finite length coal face by a coal cutting machine. During this operation, the coal cutting machine rests on the pass profile produced during the previous pass, that is, the height of the coal/stone interface above some datum line. The result can be undulations in the pass profile that increase in amplitude from pass-to-pass and require productive work to stop to enable their removal.

Applications also exist where adopting a repetitive process setting for analysis can be used to productive effect. Examples include iterative algorithms for solving nonlinear dynamic optimal control problems based on the maximum principle [Roberts (2000)]. In this case, use of the repetitive process setting provides the basis for the development of highly reliable and efficient solution algorithms. More recent work on the use of the repetitive process setting for the analysis of optimal control/optimization problems includes a gas pipeline application [Azevedo-Perdicoúlis and Jank (2012)]. Also iterative learning control algorithms can be designed in the repetitive process setting with very good agreement between predicted and experimentally measured results [Hładowski et al (2010), Hładowski et al (2011), Hładowski et al (2012)].

In some cases, the dynamics of discrete linear repetitive processes can be written in the form of a $2 \mathrm{D}$ discrete linear system described by either the Roesser [Roesser (1975)] or the Fornasini-Marchesini [Fornasini and Marchesini (1978)] state-space model. The most useful case is examples where on pass $k$ and instance $p$, written $(k, p)$, the only previous pass contribution comes from $(k-1, p)$ where a strong form of repetitive process stability is equivalent to bounded-input bounded-output stability of the Roesser or Fornasini-Marchesini state-space model representations of the dynamics. Even for these cases, however, there are important systems theoretic questions that have no equivalents for $2 \mathrm{D}$ discrete linear systems described by these state-space models [Rogers et al (2007)].

This paper considers discrete linear repetitive processes that have no Roesser or Fornasini state-space model descriptions, which arise from how the previous pass profile contributes to the current one, either in forming an adequate model of the dynamics for control law design or through application of the control law. At $(k, p)$ the vast majority of work to-date has used control laws that are actuated only by the state or pass profile vector at $(k, p)$ and pass profile vector at $(k-1, p)$. However, once pass $k$ is complete the complete pass profile vector $y_{k}(p), 0 \leq p \leq \alpha-1$ is available for use in control law design and the questions are: i) when is such a control law required and ii) how can control law design be undertaken? Moreover, application areas arise where assuming that the only previous pass contribution to the dynamics at $(k, p)$ comes from $(k-1, p)$ does not give an adequate model 
on which to base subsequent analysis. After introducing models for the case when the previous pass contribution at $(k, p)$ arises from instances other than $(k-1, p)$, from either constructing a model of the dynamics or application of a control law or both, a Lyapunov function interpretation of stability is developed, leading to Linear Matrix Inequality (LMI) based conditions that also produce formulas for the design of implementable control laws.

Throughout this paper, the null and identity matrices with the required dimensions are denoted by 0 and $I$, respectively. Also, $\bigoplus$ (and $\oplus$ ) denotes direct sum of matrices and $\otimes$ denotes the Kronecker product of matrices, $M>0(<0)$ denotes a real symmetric positive (negative) definite matrix, $X \leq Y$ is used to represent the case when $X-Y$ is a negative semi-definite matrix and $\star$ denotes a block entry in a symmetric matrix.

\section{State-space Models and Control Laws}

\subsection{Models for Repetitive Process Dynamics}

Discrete linear repetitive processes evolve over the subset of the positive quadrant in the 2D plane defined by $\{(p, k): 0 \leq p \leq \alpha-1, k \geq 0\}$, with state-space model [Rogers et al (2007)]

$$
\begin{aligned}
x_{k+1}(p+1) & =A x_{k+1}(p)+B u_{k+1}(p)+B_{0} y_{k}(p) \\
y_{k+1}(p) & =C x_{k+1}(p)+D u_{k+1}(p)+D_{0} y_{k}(p)
\end{aligned}
$$

Here on pass $k, x_{k}(p) \in \mathbb{R}^{n}$ is the state vector, $y_{k}(p) \in \mathbb{R}^{m}$ is the pass profile vector, and $u_{k}(p) \in \mathbb{R}^{r}$ is the vector of control inputs. The boundary conditions, that is, the pass state initial vector sequence and the initial pass profile, are

$$
\begin{aligned}
x_{k+1}(0) & =d_{k+1}, & & k \geq 0 \\
y_{0}(p) & =f(p), & & 0 \leq p \leq \alpha-1
\end{aligned}
$$

where the $n \times 1$ vector $d_{k+1}$ has known constant entries and $f(p)$ is an $m \times 1$ vector whose entries are known functions of $p$.

The state-space model (1) has similarities with the Roesser [Roesser (1975)] and Fornasini-Marchesini [Fornasini and Marchesini (1978)] state-space models for $2 \mathrm{D}$ discrete linear systems. These similarities have led to the use of results from the extensive literature for these models to solve systems theoretic questions for examples described by (1) and (2). There are, however, important systems theoretic questions for these processes which cannot be answered in this way. For example, pass profile controllability requires that for given boundary conditions there exists a control input sequence can be constructed and implemented such that an example described by (1) and (2) produces a pre-defined pass profile vector either on some pass or with the pass number also pre-defined. This property is well defined in terms of applications and has no 2D Roesser or Fornasini-Marchesini state-space model interpretation, nor can conditions for its existence be established using systems theory for these models.

As discussed in the previous section, longwall coal cutting can be modeled as a repetitive process. In this application, the pass profile is the height of the stone/coal 


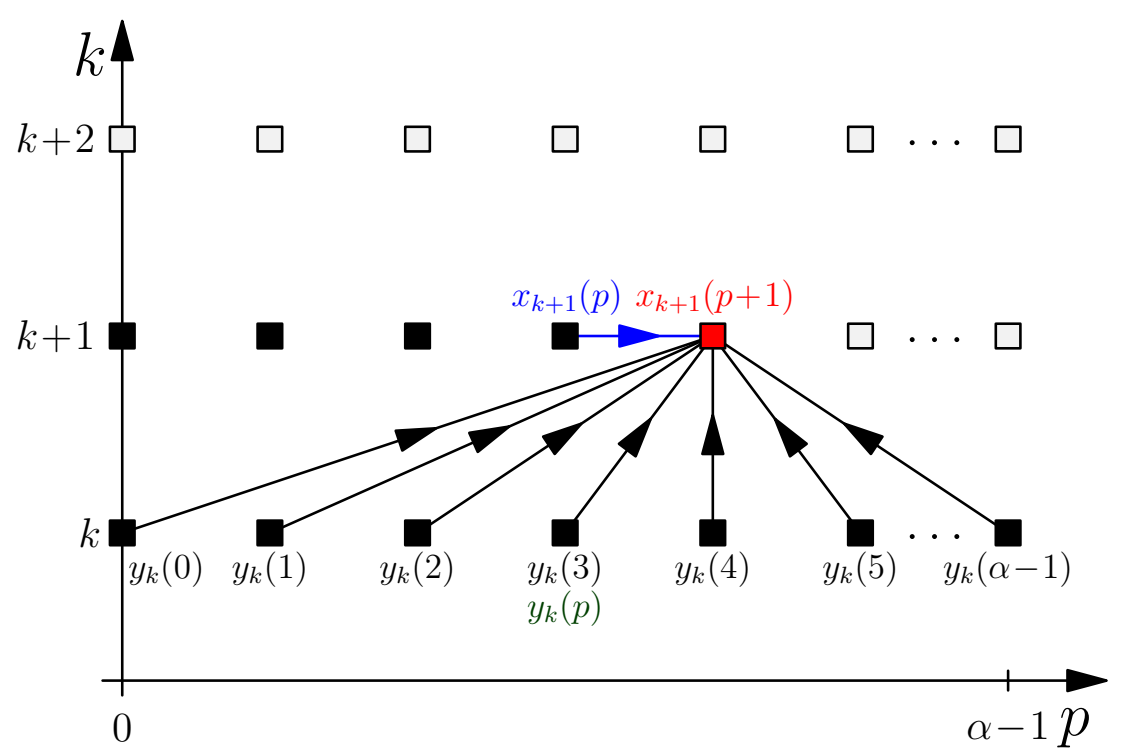

Fig. 1 Illustrating the updating of the current pass state vector structure in (3).

interface above some datum line. Also the cutting machine rests on the previous pass profile during the production of the current pass profile. As the machines used in this application area can be up to 5 tonnes in weight, it is unrealistic to employ the model of (1) and (2). The reason is that the coal cutting machine rests on top of the previous pass profile during the production of the current one and the squashing effect on the previous pass profile makes a model of the form (1 unrelaistic.. An alternative in this case is to use a model [Rogers et al (2007), Cichy et al (2010)] of the following form over $k \geq 0$ and $0 \leq p \leq \alpha-1$

$$
\begin{aligned}
x_{k+1}(p+1) & =A x_{k+1}(p)+\hat{B} u_{k+1}(p)+\sum_{i=0}^{\alpha-1} B_{i} y_{k}(i)+E y_{k}(p) \\
y_{k+1}(p) & =C x_{k+1}(p)+\hat{D} u_{k+1}(p)+\sum_{i=0}^{\alpha-1} D_{i} y_{k}(i)+F y_{k}(p)
\end{aligned}
$$

with the same notation and boundary conditions as (1) and (2). The dynamics of (3) and, in particular, the updating structure can be visualized as shown in Figs. 1 and 2, and the differences with (1) is apparent.

In this last state-space model the complete previous pass profile explicitly contributes to the state and pass profile computation at any sample instant on the current pass. This is known as interpass smoothing and the state-space model (3) and (2) will play a critical role in the analysis to follow in this paper. A model of this form has no 2D Roesser or Fornasini-Marchesini state-space model interpretations. 


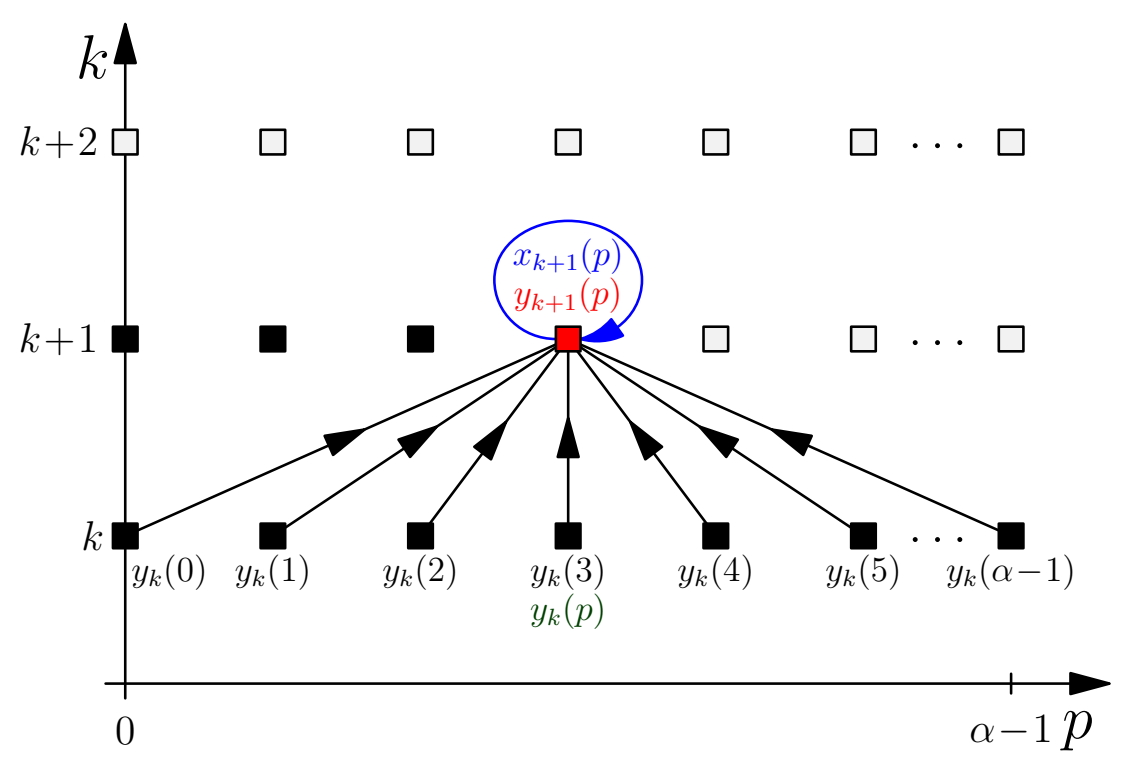

Fig. 2 Illustrating the updating of the current pass profile vector in (3).

Another model for discrete linear repetitive process dynamics is of the form

$$
\begin{aligned}
x_{k+1}(p+1) & =A x_{k+1}(p)+\hat{B} u_{k+1}(p)+\sum_{i=-w_{l}}^{w_{h}} B_{i} y_{k}(p+i) \\
y_{k+1}(p) & =C x_{k+1}(p)+\hat{D} u_{k+1}(p)+\sum_{i=-w_{l}}^{w_{h}} D_{i} y_{k}(p+i)
\end{aligned}
$$

where $w_{l}$ and $w_{h}$ are positive integers and the boundary conditions are again taken as (2), with the additional assumptions that $y_{k}(j)=0, j=-w_{l},-w_{l+1}, \ldots,-1$ and for $j=\alpha, \alpha+1, \ldots, \alpha-1+w_{l}$. In this model the previous pass contribution at $(k, p)$ is modeled as a linear sum of the pass profile vectors for $0 \leq p-w_{l} \leq p \leq p+w_{h}$. Setting $w_{l}=0$ and $w_{h}=0$ recovers the model of (1) but there is no link between this model and that of (3). On each pass in the model (4) the previous pass contributions at $(k, p)$ come from the 'window' of points $p-w_{l} \leq p \leq p+w_{h}$ that moves along the pass as the dynamics are generated and also propagate from passto-pass. This has led to term 'wave' repetitive process [Gałkowski et al (2006)] to describe examples represented by this model.

The dynamics of (4) and, in particular, the updating structure can be visualized as in Figs. 3 and 4, and the difference with that of (1) and (3) is apparent.

A model of this form also has no 2D Roesser or Fornasini-Marchesini statespace model interpretations.

As shown in [Palucki et al (2012)] a model of the form

$$
\begin{gathered}
x_{k+1}(p)=A_{1} x_{k}(p-1)+A_{2} x_{k}(p)+A_{3} x_{k}(p+1) \\
+B_{1} u_{k}^{1}(p)+B_{2} u_{k}^{2}(p)+B_{b} u_{k}^{3}(p)
\end{gathered}
$$

arises in the modeling of ladder circuits and is obtained from (4) by deleting the first equation in this repetitive process model, replacing $x$ by $y$, setting $C=0$ and 


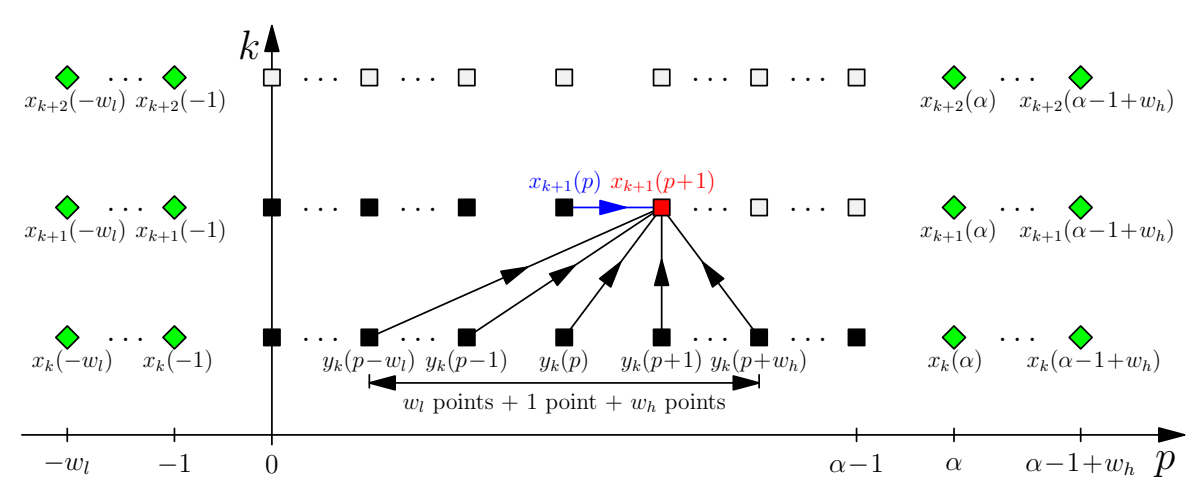

Fig. 3 Illustrating the updating structure of the current pass state vector in (4).

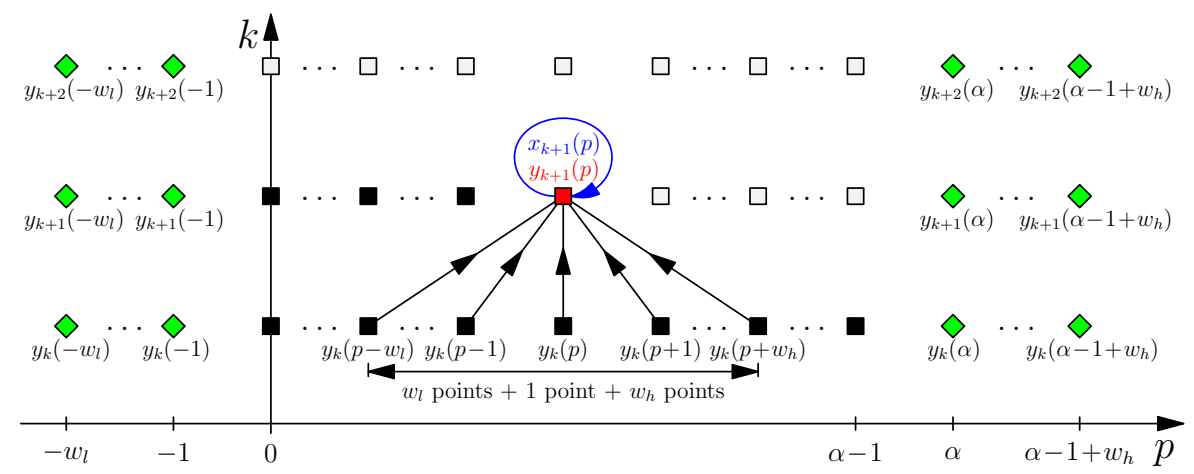

Fig. 4 Illustrating the updating structure of the current pass profile vector in (4).

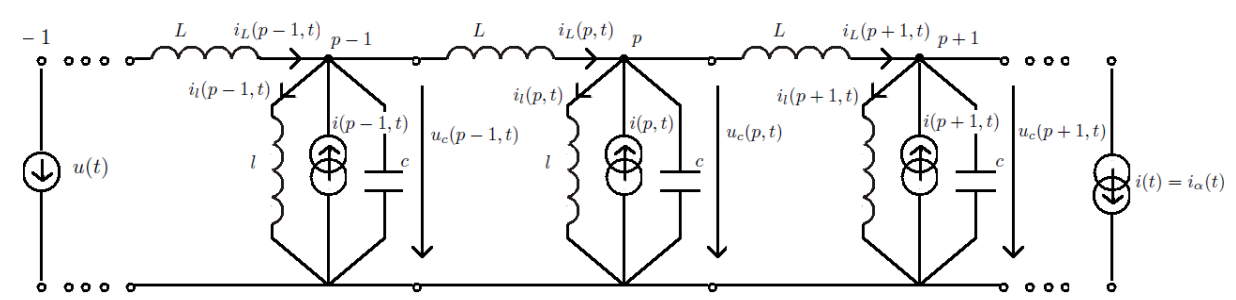

Fig. 5 A ladder circuit.

replacing $u_{k+1}$ by $u_{k}$. Consider also the ladder circuit of Fig. 5 and, in particular, its $p$-th node and define the state vector for the $p$-th ladder as

$$
x(p, t)=\left[u_{c}(p, t) i_{l}(p, t) i_{L}(p, t)\right]^{T}
$$

where $u_{c}, i_{l}, i_{L}$ denote condenser voltage and inductor currents, respectively.

The state-space model of the whole network [Palucki et al (2012)] can be written over $p=0,1, \ldots, \alpha-1$ in the form

$$
\frac{d}{d t} x(p, t)=\mathcal{A}_{1} x(p-1, t)+\mathcal{A}_{2} x(p, t)+\mathcal{A}_{3} x(p+1, t)+\mathcal{B} i(p, t)
$$


where

$$
\mathcal{A}_{1}=\left[\begin{array}{lll}
0 & 0 & 0 \\
0 & 0 & 0 \\
\frac{1}{L} & 0 & 0
\end{array}\right], \quad \mathcal{A}_{2}=\left[\begin{array}{ccc}
0 & -\frac{1}{c} & \frac{1}{c} \\
\frac{1}{l} & 0 & 0 \\
-\frac{1}{L} & 0 & 0
\end{array}\right], \quad \mathcal{A}_{3}=\left[\begin{array}{ccc}
0 & 0 & -\frac{1}{c} \\
0 & 0 & 0 \\
0 & 0 & 0
\end{array}\right], \quad \mathcal{B}=\left[\begin{array}{c}
\frac{1}{c} \\
0 \\
0
\end{array}\right]
$$

and $i(p, t), p=0,1, \ldots, \alpha-1$ represent inputs to the circuit realized as controlled current sources, that is,

$$
i(p, t)=u^{1}(p, t)+u^{2}(p, t)+u^{3}(p, t)
$$

The controls $u^{i}, i=1,2,3$ can be taken as currents or voltages as required. The boundary conditions are taken as

$$
x(-1, t)=\left[\begin{array}{c}
u(t) \\
0 \\
0
\end{array}\right], \quad x(\alpha, t)=\left[\begin{array}{c}
0 \\
0 \\
i(t)
\end{array}\right], \quad x(p, 0)=0
$$

This means that the circuit input (at node -1 ) is an autonomic voltage source and the output (at node $\alpha$ ) an autonomic current source.

Constructing a discrete representation of (7) using a direct method, such as

$$
\frac{d x(p, t)}{d t}=\frac{x(p,(k+1) \Delta T)-x(p, k \Delta T)}{\Delta T}
$$

gives a model of the form (5) where states and input are

$$
\begin{aligned}
x_{k}(p) & =\left[u_{c k}(p) i_{l k}(p) i_{L k}(p)\right]^{T} \\
i_{k}(p) & =u_{k}^{1}(p)+u_{k}^{2}(p)+u_{k}^{3}(p)
\end{aligned}
$$

respectively and

$$
A_{1}=\Delta T \mathcal{A}_{1}, \quad A_{2}=I+\Delta T \mathcal{A}_{2}, \quad A_{3}=\Delta T \mathcal{A}_{3}, \quad B_{1}=B_{2}=B_{3}=\Delta T \mathcal{B}
$$

The boundary conditions are

$$
x_{k}(-1)=\left[\begin{array}{c}
u_{k} \\
0 \\
0
\end{array}\right], \quad x_{k}(\alpha)=\left[\begin{array}{c}
0 \\
0 \\
i_{k}
\end{array}\right], \quad x(p, 0)=0
$$

where $u_{k}=u(k \Delta t)$ and $i_{k}=i(k \Delta t)$. For ease of presentation, no further explicit reference to the boundary conditions for any of the models will be made until the numerical examples in the penultimate section of this paper. 


\subsection{Control Laws}

Given the previous pass profile contribution to the current pass dynamics, control laws must be a combination of current pass feedback plus a contribution from the previous pass. For examples described by (1), suppose that all entries in the current pass state vector are available for measurement. Then one control law is of the form over $0 \leq p \leq \alpha-1, k \geq 0$

$$
u_{k+1}(p)=\left[\begin{array}{ll}
K_{1} & K_{2}
\end{array}\right]\left[\begin{array}{c}
x_{k+1}(p) \\
y_{k}(p)
\end{array}\right]
$$

where $K_{1}$ and $K_{2}$ are appropriately dimensioned matrices to be designed. This control law is composed of the weighted sum of current pass state feedback and feedforward of the previous pass profile. If $x_{k+1}(p)$ is not available then one option is to include an observer to estimate this vector and another to replace it with the current pass profile vector. It is also possible to use a control law with its own state dynamics and the standing assumption is that the levels of noise and other disturbances on the entries in the measured vectors are negligible. Control laws of these forms can also be applied to processes described by (3) and (4) but given the extra previous pass profile contributions to the current pass profile they are likely to be very weak.

It is possible that the control law (15) is unable to stabilize a process described by (1) and a more stronger form is required. Once a pass is completed, the complete previous pass profile is available for use in a control law. For example, the following control law can be defined for processes described by (1)

$$
\begin{gathered}
u_{k+1}(p)=K_{x}(p) x_{k+1}(p)+\sum_{i=0}^{\alpha-1} K_{i} y_{k}(i)+K_{y}(p) y_{k}(p) \\
=\left[\begin{array}{lllll}
K_{x}(p) & K_{0} & \cdots & K_{\alpha-1} & K_{y}(p)
\end{array}\right]\left[\begin{array}{c}
x_{k+1}(p) \\
y_{k}(0) \\
\vdots \\
y_{k}(\alpha-1) \\
y_{k}(p)
\end{array}\right]
\end{gathered}
$$

where the matrix functions $K_{x}(p)$ and $K_{y}(p)$ depend on the sampling instance $p$ along the pass, $0 \leq p \leq \alpha-1$. Again, in implementation terms, this control law requires that all elements of the current pass state vector are available for measurement. If this is not the case then an observer will be required or else the state vector must be replaced by the pass profile vector. Applying (16) to (1) results in a controlled process state-space model of the form (3).

It is also possible to replace the lower and upper limits in the sum that forms the previous pass contribution in the above control law and hence only a restrictive number of sample instances along the previous pass profile appear in the control law. Hence an alternative control law to (16) is

$$
u_{k+1}(p)=K_{x} x_{k+1}(p)+\sum_{i=-w_{l}}^{w_{h}} K_{i} y_{k}(p+i)
$$


and applying this control law to (1) results in a controlled process state-space model of the form (4). Another application for repetitive processes is the design of iterative learning control laws, the survey papers [Bristow et al (2006)], [Hyo-Sung et al (2007)] are one starting point for background on this form of control, where design in this setting offers advantages over alternatives, such as simultaneous control over both error convergence from pass-to-pass (or trial-to-trial in the iterative learning control literature) and guaranteed monotonic pass-to-pass error convergence. A control law of the form (17) arises in the case when the first Markov parameter in the system state-space model is zero [Hładowski et al (2011)].

The next section discusses the stability of the models introduced in this section as a prelude to stabilizing control law design which is treated in the following one.

\section{Stability Analysis}

The stability theory [Rogers et al (2007)] for linear repetitive processes is based on an abstract model in a Banach space setting that includes a large number of such processes as special cases, including those considered in this paper. In this setting, a bounded linear operator mapping a Banach space into itself describes the contribution of the previous pass dynamics to the current one and the stability conditions are described in terms of properties of this operator. Given the unique feature of these processes, that is, oscillations that increase in amplitude from pass-to-pass, the $k$ direction in the notation for variables used so far in this paper, this theory is based on ensuring that a bounded initial pass profile produces a bounded sequence of pass profiles generated $\left\{y_{k}\right\}$, where bounded is defined in terms of the norm on the underlying Banach space.

Two forms of stability can be defined in this setting, which are termed asymptotic stability and stability along the pass, respectively. The former requires this property with respect to the, finite and fixed, pass length and the latter uniformly, that is, independent of the pass length.

The abstract model for linear constant pass length linear repetitive processes of pass length $\alpha$ describes the process dynamics as

$$
y_{k+1}=L_{\alpha} y_{k}+b_{k+1}, k \geq 0
$$

where $y_{k} \in E_{\alpha}$ and $E_{\alpha}$ is a suitably chosen Banach space and $\|\cdot\|$ is used to denote both the norm on $E_{\alpha}$ and also the induced operator norm. Also $L_{\alpha}$ is a bounded linear operator mapping $E_{\alpha}$ intro itself and $b_{\alpha} \in W_{\alpha}$, where $W_{\alpha}$ is a linear subspace of $E_{\alpha}$. In this model the term $L_{\alpha} y_{k}$ represents the contribution of pass $k$ to pass $k+1, k \geq 0$ and $b_{k+1}$ represents terms that enter on pass $k+1$, that is, the control input and the effects of the current pass initial conditions and any disturbances acting on this pass.

Asymptotic stability of (18) requires the existence of finite real scalars $M_{\alpha}>0$ and $\lambda_{\alpha} \in(0,1)$ such that $\left\|L_{\alpha}^{k}\right\| \leq M_{\alpha} \lambda_{\alpha}^{k}, k \geq 0$, which, in turn, is equivalent to $r\left(L_{\alpha}\right)<1$ where $r(\cdot)$ denotes the spectral radius. Also if this property holds then the strong limit $y_{\infty}:=\lim _{k \rightarrow \infty} y_{k}$ is termed the limit profile and is the unique solution of the linear equation

$$
y_{\infty}=L_{\alpha} y_{\infty}+b_{\infty}
$$


In the case of processes described by (1), it is known [Rogers et al (2007)] that asymptotic stability holds if and only if all eigenvalues of the matrix $D_{0}$ lie in the open unit circle in the complex plane, that is, $r\left(D_{0}\right)<1$. If asymptotic stability holds and the control input sequence applied is strongly convergent in the passto-pass direction, the limit profile (19) is described by a $1 \mathrm{D}$ discrete linear system state-space model with state matrix $A+B_{0}\left(I-D_{0}\right)^{-1} C$ and hence can be unstable as the simple case when $A=-0.5, B_{0}=0.5+\beta, C=1, D=0$, and $D_{0}=0$, where $\beta$ is a real scalar with $|\beta| \geq 1$, demonstrates. The reason why this case arises is due to the finite pass length over which duration even an unstable standard linear system can only produce a bounded output.

To prevent examples such as the one given above from arising, stability along the pass demands the existence of finite real scalars $M_{\infty}>0$ and $\lambda_{\infty} \in(0,1)$, which are independent of $\alpha$, such that $\left\|L_{\alpha}^{k}\right\| \leq M_{\infty} \lambda_{\infty}^{k}, k \geq 0$. In the case of processes described by (1), it can be shown [?] that necessary and sufficient conditions for stability along the pass of processes described by the state-space model (1) are

i) $r\left(D_{0}\right)<1$,

ii) $r(A)<1$

iii) all eigenvalues of the transfer-function matrix

$$
G(z)=C(z I-A)^{-1} B_{0}+D_{0}
$$

have modulus strictly less than unity for all $|z|=1$.

Each of these conditions has a well defined physical interpretation and for the simple example given above it is condition iii) that does not hold when $|\beta| \geq$ 1. Moreover, each of these conditions has a well defined physical interpretation but the interpretation of these conditions for processes described by the other state-space models considered in this paper does not have the same transparency. Consequently control law design is addressed using a Lyapunov function approach, leading to algorithms that can be computed using LMIs.

Consider the following Lyapunov function for processes described by (1)

$$
V(k, p)=V_{1}(k, p)+V_{2}(k, p)
$$

where

$$
\begin{aligned}
& V_{1}(k, p)=x_{k+1}^{T}(p) P x_{k+1}(p) \\
& V_{2}(k, p)=y_{k}^{T}(p) Q y_{k}(p)
\end{aligned}
$$

where $P>0$ and $Q>0$. The term $V_{1}(k)$ captures the current pass state vector energy and $V_{2}(k)$ the previous pass profile energy. Also introduce the increment of $V(k, p)$ as

$$
\Delta V(k, p)=V_{1}(k, p+1)-V_{1}(k, p)+V_{2}(k+1, p)-V_{2}(k, p)
$$

Then it has been shown [Rogers et al (2007)] that processes described by (1) are stable along the pass if

$$
\Delta V(k, p)<0
$$

In the case of processes described by (3) introduce the Lyapunov function

$$
V(k)=V_{1}(k)+V_{2}(k)
$$


where

$$
V_{1}(k)=\sum_{i=0}^{\alpha-1} y_{k}^{T}(i) Q_{i} y_{k}(i), \quad V_{2}(k)=\sum_{i=0}^{\alpha-1} x_{k}^{T}(i) P_{i} x_{k}(i)
$$

where $Q_{i}>0, i=0,1, \ldots, \alpha-1$ and $P_{i}>0, i=0,1, \ldots, \alpha$. The term $V_{1}(k)$ captures the pass-to-pass energy change and $V_{2}(k)$ the change in energy along a pass. Also introduce

$$
V_{2}^{\prime}(k)=\sum_{i=1}^{\alpha} x_{k}^{T}(i) P_{i} x_{k}(i)
$$

and define the increment of this Lyapunov function as

$$
\Delta V(k)=V_{1}(k+1)-V_{1}(k)+V_{2}^{\prime}(k+1)-V_{2}(k+1)
$$

together with the notation

$$
\begin{gathered}
\widehat{A}=I \otimes A, \quad \widehat{C}=I \otimes C, \quad \widehat{E}=I \otimes E, \quad \widehat{F}=I \otimes F \\
\widehat{Q}=\bigoplus_{i=0}^{\alpha-1} Q_{i}, \quad \widehat{P}_{1}=\bigoplus_{i=0}^{\alpha-1} P_{i}, \quad \widehat{P}_{2}=\bigoplus_{i=1}^{\alpha} P_{i}
\end{gathered}
$$

and

$$
\widetilde{B}=\left[\begin{array}{ccc}
B_{0} & \cdots & B_{\alpha-1} \\
\vdots & \ddots & \vdots \\
B_{0} & \cdots & B_{\alpha-1}
\end{array}\right], \quad \widetilde{D}=\left[\begin{array}{ccc}
D_{0} & \cdots & D_{\alpha-1} \\
\vdots & \ddots & \vdots \\
D_{0} & \cdots & D_{\alpha-1}
\end{array}\right]
$$

Then we have the following result.

Theorem 1 [Cichy et al (2010)] A discrete linear repetitive process described by (3) is stable along the pass if there exist matrices $\tilde{Q}_{i}>0, i=0,1, \ldots, \alpha-1$, and $\tilde{P}_{i}>0$, $i=0,1, \ldots, \alpha$, such that the following LMI holds

$$
\left[\begin{array}{cc}
-\mathcal{P}_{1} & \mathcal{P}_{1} \mathcal{A}^{T} \\
\mathcal{A} \mathcal{P}_{1} & -\mathcal{P}_{2}
\end{array}\right]<0
$$

where

$$
\mathcal{P}_{1}=\bar{P}_{1} \oplus \bar{Q}, \quad \mathcal{P}_{2}=\bar{P}_{2} \oplus \bar{Q}, \quad \mathcal{A}=\left[\begin{array}{ll}
\widehat{A} & \widetilde{B}+\widehat{E} \\
\widehat{C} & \widetilde{D}+\widehat{F}
\end{array}\right]
$$

and

$$
\bar{Q}=\bigoplus_{i=0}^{\alpha-1} \tilde{Q}_{i}, \quad \bar{P}_{1}=\bigoplus_{i=0}^{\alpha-1} \tilde{P}_{i}, \quad \bar{P}_{2}=\bigoplus_{i=1}^{\alpha} \tilde{P}_{i}
$$

In the case of processes described by (4), consider the Lyapunov function

$$
V(k, p)=V_{1}(k, p)+V_{2}(k, p)
$$

where

$$
V_{1}(k, p)=\sum_{i=-w_{l}}^{w_{h}} y_{k}^{T}(p+i) Q_{i} y_{k}(p+i)
$$


and

$$
V_{2}(k, p)=x_{k}^{T}(p) P x_{k}(p)
$$

where $P>0$ and $Q_{i}>0, i=-w_{l}, \ldots, 0, \ldots, w_{h}$. The term $V_{1}(k, p)$ captures the pass-to-pass energy change and $V_{2}(k, p)$ the change in energy along a pass. Moreover, the associated increment is

$$
\begin{aligned}
\Delta V(k, p) & =y_{k+1}^{T}(p)\left(\sum_{i=-w_{l}}^{w_{h}} Q_{i}\right) y_{k+1}(p)+x_{k+1}^{T}(p+1) P x_{k+1}(p+1) \\
& -\sum_{i=-w_{l}}^{w_{h}} y_{k}^{T}(p+i) Q_{i} y_{k}(p+i)-x_{k+1}^{T}(p) P x_{k+1}(p)
\end{aligned}
$$

Summing over $p=0,1, \ldots, \alpha$ gives the global Lyapunov function

$$
V(k)=\sum_{p=0}^{\alpha} V(k, p)
$$

with associated increment

$$
\Delta V(k)=\sum_{p=0}^{\alpha} \Delta V(k, p)
$$

The proof of the next result follows by routine extensions to that for a process described by (1) given in [Rogers et al (2007)] and hence the details are omitted.

Theorem 2 A discrete linear repetitive process described by (4) is stable along the pass if

$$
\Delta V(k)<0
$$

where $\Delta V(k)$ is given by (38).

\section{Stabilization}

Consider first application of the control law (15) to processes described by (1). Then the following result gives a sufficient condition for stability along the pass of the resulting controlled process with formulas for designing the stabilizing control law matrices

Theorem 3 [Rogers et al (2007)] Suppose that a control law of the form (15) is applied to a discrete linear repetitive process described by (1). Then the resulting controlled process is stable along the pass if there exist matrices $W=\operatorname{diag}\left(W_{1}, W_{2}\right)$, $W_{1}>0, W_{2}>0, G$, and

$$
N=\left[\begin{array}{ll}
\bar{N}_{1} & \bar{N}_{2} \\
\bar{N}_{1} & \bar{N}_{2}
\end{array}\right]
$$

such that

$$
\left[\begin{array}{cc}
-G-G^{T}+W & (\Phi G+\widehat{B} N)^{T} \\
\Phi G+\widehat{B} N & -W
\end{array}\right]<0
$$


where $\Phi$ denotes the augmented system matrix

$$
\Phi=\left[\begin{array}{ll}
A & B_{0} \\
C & D_{0}
\end{array}\right]
$$

If the LMI of (41) is feasible, stabilizing $K_{1}$ and $K_{2}$ in the control law (15) are given by

$$
K=N G^{-1}
$$

with

$$
K=\left[\begin{array}{ll}
K_{1} & K_{2} \\
K_{1} & K_{2}
\end{array}\right], \quad \widehat{B}=\left[\begin{array}{cc}
B & 0 \\
0 & D
\end{array}\right]
$$

The conditions of Theorem 3 are sufficient only and hence there is an associated level of conservativeness, which means that if this theorem does not hold for a given example a stabilizing control law may exist. Hence there is a need to investigate ways of reducing this level of conservativeness and this problem is considered in the remainder of this section. The results developed are supported by two design examples in the next section.

Applying the control law (16) to (1) gives the controlled process state-space model

$$
\begin{aligned}
x_{k+1}(p+1) & =\left(A+B K_{x}(p)\right) x_{k+1}(p)+\sum_{i=0}^{\alpha-1} B K_{i} y_{k}(i)+\left(B_{0}+B K_{y}(p)\right) y_{k}(p) \\
y_{k+1}(p) & =\left(C+D K_{x}(p)\right) x_{k+1}(p)+\sum_{i=0}^{\alpha-1} D K_{i} y_{k}(i)+\left(D_{0}+D K_{y}(p)\right) y_{k}(p)
\end{aligned}
$$

which is of the form of the state-space model (3) for a discrete linear repetitive process with interpass smoothing.

To apply the result of Theorem 1, introduce the notation

$$
\begin{gathered}
\widehat{B}=I \otimes B, \quad \widehat{D}=I \otimes D \\
\widehat{K}_{x}=\bigoplus_{p=0}^{\alpha-1} K_{x}(p), \quad \widehat{K}_{y}=\bigoplus_{p=0}^{\alpha-1} K_{y}(p), \quad \widehat{K}=\bigoplus_{i=0}^{\alpha-1} K_{i} \\
\widehat{N}_{x}=\bigoplus_{p=0}^{\alpha-1} N_{x}(p), \quad \widehat{N}_{y}=\bigoplus_{p=0}^{\alpha-1} N_{y}(p), \quad \widehat{N}=\bigoplus_{i=0}^{\alpha-1} N_{i} \\
\bar{B}=\left[\begin{array}{ccc}
B & \cdots & B \\
\vdots & \ddots & \vdots \\
B & \cdots & B
\end{array}\right], \quad \bar{D}=\left[\begin{array}{ccc}
D & \cdots & D \\
\vdots & \ddots & \vdots \\
D & \cdots & D
\end{array}\right]
\end{gathered}
$$

together with

$$
\begin{array}{ll}
\mathcal{A}_{K}=\widehat{A}+\widehat{B} \widehat{K}_{x}, & \mathcal{E}_{K}=\widehat{B_{0}}+\widehat{B} \widehat{K}_{y} \\
\mathcal{C}_{K}=\widehat{C}+\widehat{D} \widehat{K}_{x}, & \mathcal{F}_{K}=\widehat{D_{0}}+\widehat{D} \widehat{K}_{y} \\
\mathcal{B}_{K}=\bar{B} \widehat{K}, & \mathcal{D}_{K}=\bar{D} \widehat{K}
\end{array}
$$


and

$$
\begin{aligned}
& \mathcal{X}=\mathcal{B}_{K}+\mathcal{E}_{K}=\bar{B} \widehat{K}+\widehat{B_{0}}+\widehat{B} \widehat{K}_{y} \\
& \mathcal{Y}=\mathcal{D}_{K}+\mathcal{F}_{K}=\bar{D} \widehat{K}+\widehat{D_{0}}+\widehat{D} \widehat{K}_{y}
\end{aligned}
$$

Then (30) applied to this case can be written as

$$
\left[\begin{array}{cccc}
-\bar{P}_{1} & 0 & \bar{P}_{1} \mathcal{A}^{T} & \bar{P}_{1} \mathcal{C}^{T} \\
0 & -\bar{Q} & \bar{Q} \mathcal{X}^{T} & \bar{Q}^{T} \\
\mathcal{A} \bar{P}_{1} & \mathcal{X} \bar{Q} & -\bar{P}_{2} & 0 \\
\mathcal{C} \bar{P}_{1} & \mathcal{Y} \bar{Q} & 0 & -\bar{Q}
\end{array}\right]<0
$$

or

$$
\left[\begin{array}{cccc}
-\bar{P}_{1} & \star & \star & \star \\
0 & -\bar{Q} & \star & \star \\
\widehat{A} \bar{P}_{1}+\widehat{B} \widehat{K}_{x} \bar{P}_{1} & \bar{B} \widehat{K} \bar{Q}+\widehat{B_{0}} \bar{Q}+\widehat{B} \widehat{K}_{y} \bar{Q} & -\bar{P}_{2} & \star \\
\widehat{C} \bar{P}_{1}+\widehat{D} \widehat{K}_{x} \bar{P}_{1} & \bar{D} \widehat{K} \bar{Q}+\widehat{D_{0}} \bar{Q}+\widehat{D} \widehat{K}_{y} \bar{Q} & 0 & -\bar{Q}
\end{array}\right]<0
$$

where $\widehat{B_{0}}=I \otimes B_{0}$ and $\widehat{D_{0}}=I \otimes D_{0}$.

This last condition is not in LMI form and hence there are no effective computational methods available to compute the control law matrices. The following result removes this difficulty.

Theorem 4 Suppose that a control law of the form (16) is applied to a discrete linear repetitive process described by (1). Then the resulting controlled process is stable along the pass if there exist matrices $\tilde{Q}_{i}>0, i=0,1, \ldots, \alpha-1, \tilde{P}_{i}>0, i=0,1, \ldots, \alpha$, $N_{x}(p), N_{y}(p)$, and $N_{i}, p, i=0,1, \ldots, \alpha-1$, such that the following LMI is feasible

$$
\left[\begin{array}{cccc}
-\bar{P}_{1} & \star & \star & \star \\
0 & -\bar{Q} & \star & \star \\
\widehat{A} \bar{P}_{1}+\widehat{B} \widehat{N}_{x} & \bar{B} \widehat{N}+\widehat{B_{0}} \bar{Q}+\widehat{B} \widehat{N}_{y} & -\bar{P}_{2} & 0 \\
\widehat{C} \bar{P}_{1}+\widehat{D} \widehat{N}_{x} & \bar{D} \widehat{N}+\widehat{D_{0}} \bar{Q}+\widehat{D} \widehat{N}_{y} & 0 & -\bar{Q}
\end{array}\right]<0
$$

If this LMI is feasible, stabilizing control law matrices are given by

$$
\widehat{K}_{x}=\widehat{N}_{x} \bar{P}_{1}^{-1}, \quad \widehat{K}_{y}=\widehat{N}_{y} \bar{Q}^{-1}, \quad \widehat{K}=\widehat{N} \bar{Q}^{-1}
$$

Proof Follows immediately on substituting

$$
\widehat{K}_{x} \bar{P}_{1}=\widehat{N}_{x}, \quad \widehat{K}_{y} \bar{Q}=\widehat{N}_{y}, \quad \widehat{K} \bar{Q}=\widehat{N}
$$

into (50) to give (51).

The previous control law uses information of the pass profile from the complete previous pass and hence, especially for large $\alpha$, numerical difficulties may result. An alternative is to use the control law (17) where only sampling instances in some 'window' around $p$ are used, resulting in the controlled process state-space model

$$
\begin{aligned}
x_{k+1}(p+1) & =\mathbb{A} x_{k+1}(p)+\sum_{\substack{i=-w_{l} \\
i \neq 0}}^{w_{h}} \mathbb{B}_{i} y_{k}(p+i)+\mathbb{B}_{0} y_{k}(p) \\
y_{k+1}(p) & =\mathbb{C} x_{k+1}(p)+\sum_{\substack{i=-w_{l} \\
i \neq 0}}^{w_{h}} \mathbb{D}_{i} y_{k}(p+i)+\mathbb{D}_{0} y_{k}(p)
\end{aligned}
$$


where

$$
\begin{array}{lll}
\mathbb{A}=A+B K_{x}, & \mathbb{B}_{i}=B K_{i}, & \mathbb{B}_{0}=B_{0}+B K_{0} \\
\mathbb{C}=C+D K_{x}, & \mathbb{D}_{i}=D K_{i}, & \mathbb{D}_{0}=D_{0}+D K_{0}
\end{array}
$$

which is of the form (4) with boundary conditions (2).

To apply the result of Theorem 2 , introduce the following notation

$$
\gamma=w_{l}+w_{h}+2
$$

and

$$
\begin{aligned}
& \widehat{Q}=\bigoplus_{i=-w_{l}}^{w_{h}} Q_{i}, \quad \widehat{Z}=P \oplus \widehat{Q}, \quad \widehat{P}=I \otimes P
\end{aligned}
$$

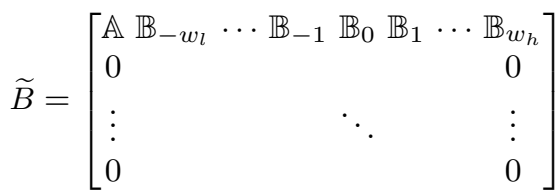

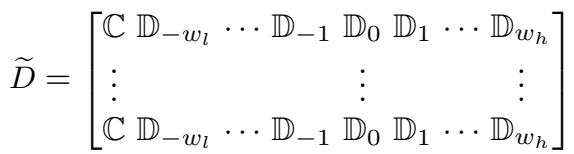

where the block entry matrices $\widetilde{B}$ and $\widetilde{D}$ are of dimensions $\gamma \times \gamma$ and $(\gamma-1) \times \gamma$ respectively. It also follows that the condition of Theorem 2 can be written as

$$
\widetilde{D}^{T} \widehat{Q} \widetilde{D}+\widetilde{B}^{T} \widehat{P} \widetilde{B}-\widehat{Z}<0
$$

This condition is not in LMI form and hence no effective methods are available to compute the control law matrices. The following result removes this difficulty.

Theorem 5 Suppose that a control law of the form (17) is applied to a discrete linear repetitive process described by (1). Then the resulting controlled process is stable along the pass if exist matrices $\check{P}>0, N_{x}, \check{Q}_{i}>0$, and $N_{i}, i=-w_{l}, \ldots, 0, \ldots, w_{h}$ such that the following LMI is feasible

$$
\left[\begin{array}{ccc}
-\bar{Z} & \star & \star \\
\widehat{A} \bar{Q}+\widehat{B} \widehat{N} & -\bar{P} & \star \\
\widehat{C} \bar{Q}+\widehat{D} \widehat{N} & 0 & -\bar{Q}
\end{array}\right]<0
$$

where

$$
\begin{aligned}
& \bar{Z}=\check{P} \oplus \bar{Q}, \quad \bar{P}=I \otimes \check{P}, \quad \check{P}=P^{-1}
\end{aligned}
$$

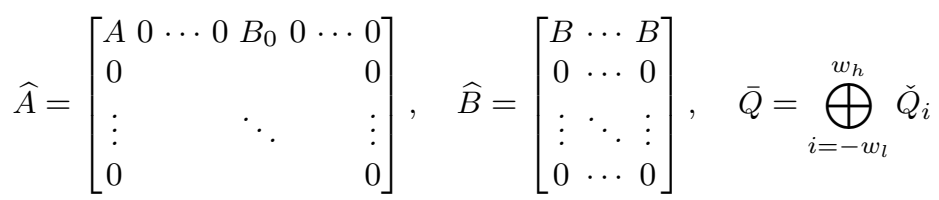

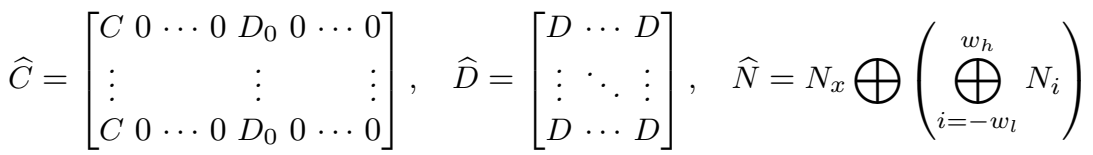


If the LMI (59) is feasible, stabilizing matrices in the control law (17) are given by

$$
K_{x}=N_{x} \check{P}^{-1}, K_{i}=N_{i} \check{Q}_{i}^{-1}, i=-w_{l}, \ldots, w_{h}
$$

Proof In the file I received it said multiply by $\operatorname{diag}\left(\widehat{Z}^{-1}, I, I\right)$ — this cannot be correct as it does not say from the right or the left - I have modified this to that below. This needs checking and if necessary correcting. Then remove this text in red.

First make two applications of the Schur's complement formula to (58), pre and post-multiply the result by $\operatorname{diag}\left(\widehat{Z}^{-1}, I, I\right)$ and introduce the variable substitution

$$
\bar{Q}=\widehat{Q}^{-1}, \quad \bar{P}=\widehat{P}^{-1}, \quad \bar{Z}=\widehat{Z}^{-1}
$$

to obtain

$$
\left[\begin{array}{ccc}
-\bar{Z} & \bar{Z} \widetilde{B}^{T} & \bar{Z} \widetilde{D}^{T} \\
\widetilde{B} \bar{Z} & -\bar{P} & 0 \\
\widetilde{D} \bar{Z} & 0 & -\bar{Q}
\end{array}\right] \prec 0
$$

where

$$
{ }^{\prime} 1 \bar{Q}=\bigoplus_{i=-\epsilon}^{\varepsilon} \check{Q}_{i}, \quad \bar{Z}=\bar{P} \oplus \bar{Q}, \quad \bar{P}=I_{\gamma} \otimes \check{P}
$$

where $P^{-1}=\check{P} \succ 0$ and $Q_{i}^{-1}=\check{Q}_{i} \succ 0, i=-w_{h}, \ldots, 0, \ldots, w_{l}$. Finally, introducing

$$
\begin{gathered}
K_{x} \check{P}=N_{x} \\
K_{i} \check{Q}_{i}=N_{i}, \quad i=-w_{l}, \ldots, w_{h}
\end{gathered}
$$

yields (59).

\section{Design Examples}

Direct application of the Theorem 4 technique can give solutions characterized by very small entries in the control law matrices $K_{i}, i=0,1, \ldots, \alpha-1$ that, in turn, leads to a very large control demands. If, however, the LMI (51) holds there are infinitely many other solutions and to obtain an appropriate set of control law matrices it is necessary to introduce an additional optimization procedure. In this work the optimization procedure used is

$$
\max \left(\sum_{i=0}^{\alpha} \operatorname{trace}\left(\tilde{P}_{i}\right)+\sum_{i=0}^{\alpha-1} \operatorname{se}\left(N_{i}\right)\right)
$$

subject to

$$
\left[\begin{array}{cccc}
-\bar{P}_{1} & \star & \star & \star \\
0 & -\bar{Q} & \star & \star \\
\widehat{A} \bar{P}_{1}+\widehat{B} \widehat{N}_{x} & \bar{B} \widehat{N}+\widehat{B_{0}} \bar{Q}+\widehat{B} \widehat{N}_{y} & -\bar{P}_{2} & 0 \\
\widehat{C} \bar{P}_{1}+\widehat{D} \widehat{N}_{x} & \bar{D} \widehat{N}+\widehat{D_{0}} \bar{Q}+\widehat{D} \widehat{N}_{y} & 0 & -\bar{Q}
\end{array}\right]<0
$$


where the function $\operatorname{se}(M)$ on an $n \times m$ matrix, say $M$, is defined as

$$
\operatorname{se}(M)=\sum_{r=1}^{n} \sum_{c=1}^{m} M(r, c)
$$

and $M(r, c)$ denotes the element in row $r$ and column $c$ of $M$.

The problem above can also arise in the application of Theorem 5. If the LMI (59) is feasible, there are infinitely many other solutions and one method of selecting an appropriate set of $K_{i}, i=-w_{l}, \ldots, w_{h}$, is to use the following optimization procedure.

$$
\max \left(\sum_{i=-w_{l}}^{w_{h}} \operatorname{trace} \check{Q}_{i}+\sum_{i=-w_{l}}^{w_{h}} \operatorname{se}\left(N_{i}\right)\right)
$$

subject to

$$
\left[\begin{array}{ccc}
-\bar{Z} & \star & \star \\
\widehat{A} \bar{Q}+\widehat{B} \widehat{N} & -\bar{P} & \star \\
\widehat{C} \bar{Q}+\widehat{D} \widehat{N} & 0 & -\bar{Q}
\end{array}\right]<0
$$

\subsection{Numerical Examples}

Consider the case of (1) with $\alpha=31$ and

$$
\begin{aligned}
& A=\left[\begin{array}{cc}
0.19 & 1.62 \\
0.76 & 0.4
\end{array}\right], \quad B=\left[\begin{array}{l}
-0.09 \\
-1.28
\end{array}\right], \quad B_{0}=\left[\begin{array}{l}
0.69 \\
0.53
\end{array}\right] \\
& C=[-1.48-1.26], \quad D=-1.63, \quad D_{0}=1.17
\end{aligned}
$$

with boundary conditions

$$
\begin{aligned}
x_{k+1}(0)=0, & k \geq 0 \\
y_{0}(p)=1, & 0 \leq p \leq \alpha-1
\end{aligned}
$$

In this case the conditions of Theorems 3 and 5 do not hold and hence it is not possible to find a stabilizing control laws using these results. The conditions of Theorem 4 do hold and the value resulting from maximizing the cost function (63) is one. The corresponding matrices in the control law (16) are listed in Table 5.1.

Figure 6 shows the sequence of pass profiles generated by the controlled process over 50 passes and Fig. 7 the corresponding control signals. These are acceptable initial designs. In applications terms, however, the control law in this example is somewhat complex and this problem will increase with the dimension of the state vector.

As a second example, consider the case of (1) with $\alpha=31$ and

$$
\begin{aligned}
A & =\left[\begin{array}{cc}
-0.59 & -0.77 \\
-0.77 & 0.09
\end{array}\right], & B=\left[\begin{array}{c}
-0.1 \\
-0.23
\end{array}\right], & B_{0}=\left[\begin{array}{l}
0.31 \\
0.47
\end{array}\right], \\
C & =\left[\begin{array}{ll}
-0.591 .18
\end{array}\right], & D=1.22, & D_{0}=-0.56
\end{aligned}
$$




\begin{tabular}{rccc}
\hline \multicolumn{3}{c}{ Control law matrices } \\
\cline { 2 - 4 }$i$ & $K_{i}$ & $K_{x}(i)$ & $K_{y}(i)$ \\
\hline 0 & $9.9107 \times 10^{-7}$ & {$[0.47970 .7629]$} & 0.6847 \\
1 & $6.3563 \times 10^{-6}$ & {$[0.53080 .9256]$} & 0.7298 \\
2 & $-1.2454 \times 10^{-6}$ & {$[0.52320 .9583]$} & 0.7477 \\
3 & $-5.9614 \times 10^{-6}$ & {$[0.51830 .9735]$} & 0.7571 \\
4 & $-8.4821 \times 10^{-6}$ & {$[0.51520 .9828]$} & 0.7631 \\
5 & $-1.083 \times 10^{-5}$ & {$[0.51290 .9887]$} & 0.7673 \\
6 & $-1.3167 \times 10^{-5}$ & {$[0.51110 .9928]$} & 0.7705 \\
7 & $-1.5274 \times 10^{-5}$ & {$[0.50950 .9956]$} & 0.7729 \\
8 & $-1.6925 \times 10^{-5}$ & {$[0.50820 .9976]$} & 0.7748 \\
9 & $-1.8177 \times 10^{-5}$ & {$[0.5070 .999]$} & 0.7763 \\
10 & $-1.9301 \times 10^{-5}$ & {$[0.5061 .0001]$} & 0.7776 \\
11 & $-2.0518 \times 10^{-5}$ & {$[0.50521 .0009]$} & 0.7786 \\
12 & $-2.1828 \times 10^{-5}$ & {$[0.50441 .0017]$} & 0.7795 \\
13 & $-2.3059 \times 10^{-5}$ & {$[0.50381 .0023]$} & 0.7802 \\
14 & $-2.3924 \times 10^{-5}$ & {$[0.50331 .0027]$} & 0.7808 \\
15 & $-2.4092 \times 10^{-5}$ & {$[0.50261 .0029]$} & 0.7813 \\
16 & $-2.3415 \times 10^{-5}$ & {$[0.50181 .0027]$} & 0.7817 \\
17 & $-2.2273 \times 10^{-5}$ & {$[0.50081 .0021]$} & 0.7821 \\
18 & $-2.1328 \times 10^{-5}$ & {$[0.49981 .0014]$} & 0.7825 \\
18 & $-2.0833 \times 10^{-5}$ & {$[0.49891 .0007]$} & 0.7829 \\
20 & $-2.0591 \times 10^{-5}$ & {$[0.49821 .0001]$} & 0.7831 \\
21 & $-2.0068 \times 10^{-5}$ & {$[0.49750 .9995]$} & 0.783 \\
22 & $-1.8394 \times 10^{-5}$ & {$[0.49620 .998]$} & 0.7826 \\
23 & $-1.5326 \times 10^{-5}$ & {$[0.4940 .9952]$} & 0.782 \\
24 & $-1.2414 \times 10^{-5}$ & {$[0.49150 .9915]$} & 0.7814 \\
25 & $-1.0159 \times 10^{-5}$ & {$[0.48930 .9887]$} & 0.7812 \\
26 & $-7.7009 \times 10^{-6}$ & {$[0.48850 .9859]$} & 0.7797 \\
27 & $-3.6404 \times 10^{-6}$ & {$[0.47880 .9759]$} & 0.779 \\
28 & $-9.928 \times 10^{-7}$ & {$[0.43670 .9123]$} & 0.7733 \\
29 & $-6.5606 \times 10^{-6}$ & {$[0.07770 .4671]$} & 0.7689 \\
30 & $-4.4439 \times 10^{-5}$ & {$[-0.6184-0.4444]$} & 0.7281 \\
\hline & & &
\end{tabular}

Table 1 The control law matrices of (16) for the first numerical example.

with boundary conditions

$$
\begin{aligned}
x_{k+1}(0)=0, & k \geq 0 \\
y_{0}(p)=1, & 0 \leq p \leq \alpha-1
\end{aligned}
$$

For this example, Theorem 3 does not produce a stabilizing control law (15) but a stabilizing control law of the form (17) with, for example, $w_{l}=5$ and $w_{h}=5$ can be designed using Theorem 5 and the optimization problem (65). The resulting control law matrices are

$$
\begin{gathered}
K_{x}=[0.4178-0.9647], K_{0}=0.1278 \\
K_{i}=-2.0242 \times 10^{-5}, i=-5, \ldots,-1,1, \ldots, 5
\end{gathered}
$$




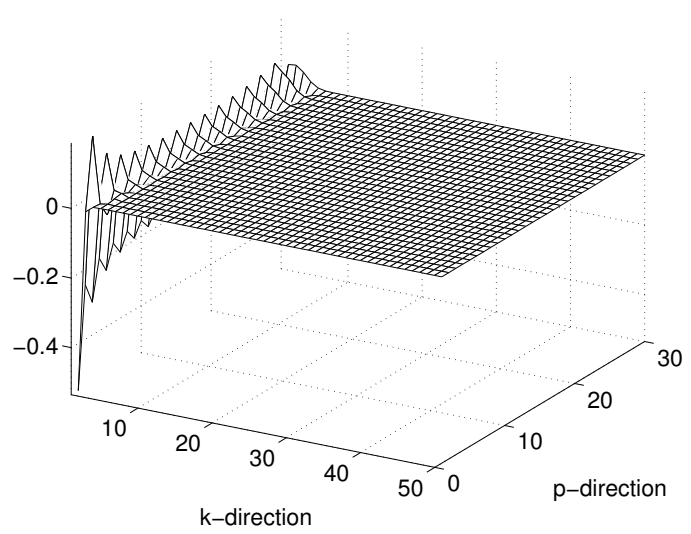

Fig. 6 Pass profiles generated by the controlled process.

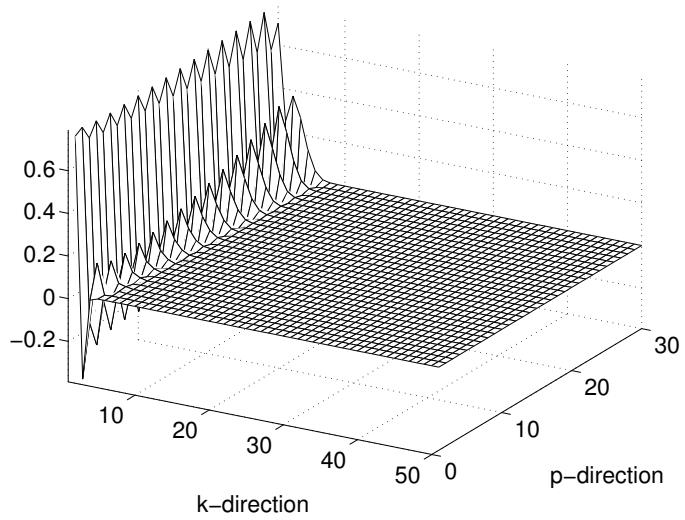

Fig. 7 Control inputs required by the controlled process.

Figs. 8 and 9, respectively, show the pass profile and control input sequences generated by the controlled process. These are acceptable but there may be a case for redesign in an attempt to reduce the values of the control signals required for the early passes. The simulations are for the case when

$$
\begin{aligned}
y_{k}(i) & =0, & -w_{l} & \leq i \leq-1 \\
y_{k}(i) & =0, & \alpha & \leq i \leq \alpha+w_{h}-1
\end{aligned}
$$

For this example, a control law of the form (16) may be found but the one given above is clearly more efficient in terms of implementation.

\section{Conclusions}

This paper has considered an extension of control laws that result in, or exploit, generalized discrete linear repetitive processes with state-space models that have a nonlocal updating structure. The structure of the previous pass profile contribution to the current pass state and pass profile dynamics takes many forms and 


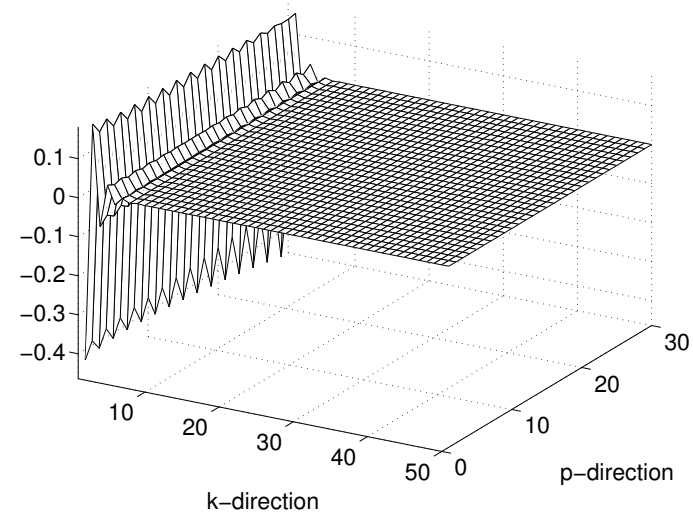

Fig. 8 Pass profiles generated by the controlled process.

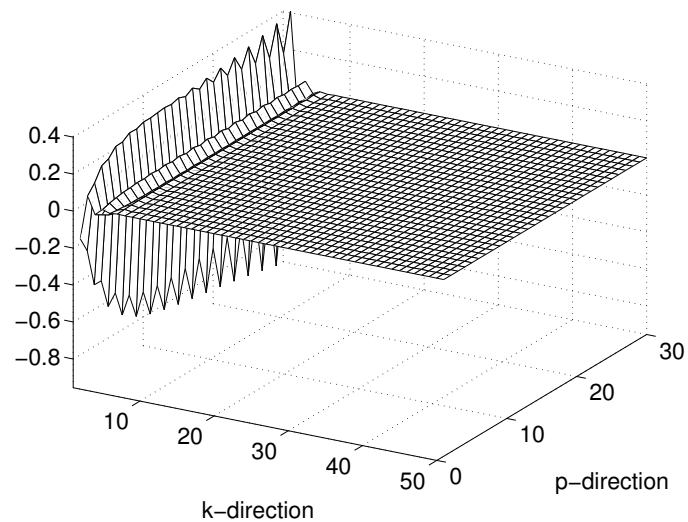

Fig. 9 Control inputs required by the controlled process.

almost all of the control law design results currently available relate to the simplest possible model structure of (1) and (2). Model structures of the form given by the state-space model (4) do have physical relevance in physical applications where its structure is one means of, at least for initial studies, including the effects of the cutting machine in the longwall coal example. The wave repetitive process model arises in the iterative learning control application and further research should aim to maximize the potential with supporting experimental verification.

A major reason for using control laws with a non-local updating structure is to reduce the conservativeness present in LMI based design of a stabilizing control law. The method is to augment the original control law with extra contributions from the previous pass profile and the new results in this paper enable control law design for stabilization. Further research is required to extend the resulting algorithms to also include performance. Other ongoing work includes replacing the current pass state vector component in the control law by a current pass profile term since the law used in this paper would require an observer unless all current pass state vector terms are directly measurable. 
The results in this paper can be extended to many other analysis and control law design problems for discrete linear repetitive processes. An example is the optimal robust control and filtering problem [Wu L. et al (2008), Wu L. et al (2009)] under various norms.

\section{References}

[Azevedo-Perdicoúlis and Jank (2012)] Azevedo-Perdicoúlis TP, Jank G (2012) Disturbance attenuation of linear quadratic OL-Nash games on repetitive processes with smoothing on the gas dynamics. Multidimensional Systems and Signal Processing 23(1-2), 131-153.

[Bristow et al (2006)] Bristow DA, Tharayil M, Alleyne A (2006) A survey of iterative learning control. IEEE Control Systems Magazine 26(3), 96-114.

[Cichy et al (2010)] Cichy B, Gałkowski K, Rogers E (2010) Control laws for discrete linear repetitive processes with smoothed previous pass dynamics, Operator Theory: Advances and Applications, vol 203, Birkhäuser Verlag Basel/Switzerland, 175-193.

[Fornasini and Marchesini (1978)] Fornasini E, Marchesini G (1978) Doubly indexed dynamical systems: state-space models and structural properties. Theory of Computing Systems $12(1), 59-72$.

[Gałkowski et al (2006)] Gałkowski K, Cichy B, Rogers E, Lam J (2006) Stabilization of a class of uncertain "wave" discrete linear repetitive processes. In: Proceedings of the 45th IEEE Conference on Decision and Control, San Diego, 1435-1440.

[Hładowski et al (2010)] Hładowski Ł, Gałkowski K, Cai Z, Rogers E, Freeman CT, Lewin PL (2010) Experimentally supported 2D systems based iterative learning control law design for error convergence and performance. Control Engineering Practice 18(4), 339-348.

[Hładowski et al (2011)] Hładowski Ł, Gałkowski K, Cai Z, Rogers E, Freeman CT, Lewin PL (2011) A 2D systems approach to iterative learning control for discrete linear processes with zero Markov parameters. International Journal of Control 84(7), 1246-1262.

[Hładowski et al (2012)] Hładowski E, Gałkowski K, Cai Z, Rogers E, Freeman CT, Lewin PL (2012) Output information based iterative learning control law design with experimental verification. Journal of Dynamic Systems, Measurement, and Control 134(2):1-10.

[Hyo-Sung et al (2007)] Hyo-Sung A, Chen Yangquan, Moore KL (2007) Iterative learning control: brief survey and categorization. IEEE Transactions on Systems Man and Cybernetics Part C: Applications and Reviews 37(6):1099-1121.

[Palucki et al (2012)] Palucki B, Gałkowski K, Kummert A, Cichy B (2012) Wave repetitive process approach to a class of ladder circuits. In: Proceedings of IEEE International Symposium on Circuits and Systems (ISCAS), In press.

[Roberts (2000)] Roberts PD (2000) Numerical investigations of a stability theorem arising from 2-dimensional analysis of an iterative optimal control algorithm. Multidimensional Systems and Signal Processing 11 (1/2), 109-124.

[Roesser (1975)] Roesser RP (1975) A discrete state-space model for linear image processing. IEEE Transactions on Automatic Control 20(1):1-10.

[Rogers et al (2007)] Rogers E, Gałkowski K, Owens DH (2007) Control Systems Theory and Applications for Linear Repetitive Processes, Lecture Notes in Control and Information Sciences, vol 349. Springer-Verlag, Berlin.

[Wu L. et al (2008)] Wu L, Lam J, Paszke W, Gałkowski K, Rogers E (2008) Robust $L_{2}-L_{\infty}$ control of uncertain differential linear repetitive processes. Int. J. Adaptive Control and Signal Processing, 22, 243-265.

[Wu L. et al (2009)] Wu L, Lam J, Paszke W, Gałkowski K, Rogers E, Kummert A (2009) Control and filtering for discrete linear repetitive processes with $H_{\infty}$ and $l_{2}-l_{\infty}$ performance. Multidimensional Systems and Signal Processing, 20(3):235-264. 